\title{
New insights into the neurological effects of COVID-19
}

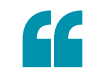

Together, these studies provide clear evidence of CNS damage in patients with COVID-19

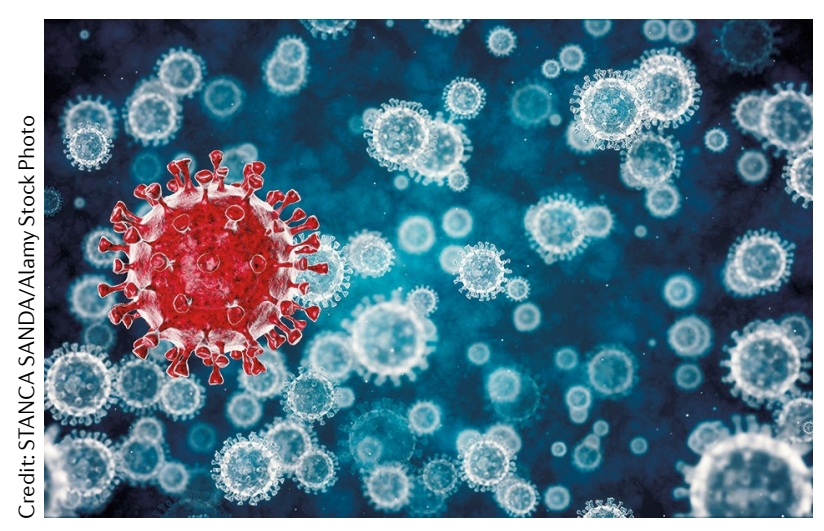

an interdisciplinary team interested in studying and treating CNS infections."

Neuropathological analysis of the brain tissue uncovered changes consistent with hypoxic injury. However, immunohistochemistry and RT-qPCR revealed minimal evidence of SARS-CoV-2 infection. The authors concluded that the hypoxic changes could be attributable to patient-specific comorbidities or secondary effects of viral infection elsewhere in the body.

The second study, published in Neurology, was led by Xavier De Tiège at the Université libre de Bruxelles, Belgium. "We wanted to investigate the occurrence of brain lesions and abnormalities with MRI in patients with severe COVID-19," explains De Tiège. "Given the practical difficulties of studying such medically unstable patients, we decided to focus on patients who had died from COVID-19."

The researchers performed brain MRI scans on 19 patients with COVID-19 within $24 \mathrm{~h}$ of death. Parenchymal brain MRI abnormalities, including white matter changes, posterior reversible encephalopathy syndrome and haemorrhage, were detected in four individuals. The researchers suggested that these changes could have resulted from blood-brain barrier breakdown and did not necessarily imply direct SARS-CoV-2 infection of brain cells.

De Tiège and colleagues also found evidence of olfactory bulb asymmetry - a possible MRI correlate of anosmia - in four patients. No brainstem changes were evident, casting doubt on suggestions of brain involvement in COVID-19-related respiratory distress.
The third study, also published in Neurology, used blood-based biomarkers to look for evidence of CNS damage in patients with COVID-19. A team led by Magnus Gisslén in Gothenburg, Sweden measured plasma levels of glial fibrillary acidic protein (GFAP) and neurofilament light chain (NfL) markers of astrocytic and neuronal injury, respectively - in 47 patients with COVID-19.

In patients with severe COVID-19 $(n=18)$, levels of both biomarkers were initially elevated; however, GFAP levels subsequently declined while NfL levels continued to increase. Plasma GFAP levels were also raised in patients with moderate COVID-19 $(n=9)$.

"These findings possibly reflect a sequence of early astrocytic response and more delayed axonal injury," suggests Gisslén. "An important outstanding question relates to the pathogenesis behind the CNS injury in COVID-19: is it related to the profound immune activation linked to systemic SARS-CoV-2 infection, and do hypoxic or ischaemic CNS events have an impact?"

Together, these studies provide clear evidence of CNS damage in patients with COVID-19. However, further research will be required to determine whether the neurological manifestations of COVID-19 involve direct viral infection of brain tissue by SARS-CoV-2.

Heather Wood

ORIGINAL ARTICLES Solomon, I. H. et al. Neuropathological features of Covid-19. N. Engl. J.Med. https://doi.org/10.1056/NEJMc2019373 (2020)|Coolen, T. et al. Early postmortem brain MRI findings in COVID-19 non-survivors. Neurology https://doi.org/10.1212/WNL.0000000000010116 (2020)| Kanberg, N. et al. Neurochemical evidence of astrocytic and neuronal injury commonly found in COVID-19. Neurology https://doi.org/10.1212/ WNL.0000000000010111 (2020) 